\title{
Performance Research on the Cost Control of Third Party Logistics Enterprises
}

\author{
Xiaobing Pei, Mengying Liu ${ }^{\text {a }}$ \\ School of Management Tianjin University of Technology Tianjin 300384, China \\ almy8341@126.com
}

Keywords: the third party logistics enterprises; activity-based costing; cost control; performance evaluation.

Abstract. The cost control is an important problem that the logistics enterprises are faced with. This study attempts to redevise the cost and build a cost performance evaluation system within the enterprise from the cost control factors and according to activity-based costing.

\section{Introduction}

The third party logistics refers to a business model [1] that the logistics service is provided by the logistics enterprises except the supply side and the demand side. Because the third party logistics enterprises have already had the activity-based costing application conditions [2], how to make a scientific and comprehensive analysis and evaluation on the performance of the third party logistics enterprise cost control has become a subject for the third party logistics cost control to solve urgently.

\section{The Third Party Logistics}

The third party logistics came into being accompanied by the deepening of the thought of supply chain management. In the nineteen nineties, the enterprises began to establish a strategic alliance and they formed a strategic partnership. On the one hand, the members of strategic alliance of enterprises paid most attention to the core business, with a view to breed the core competition ability; in this case, the members who had competitive advantage on logistics management developed into an operation mode of third party logistics with integrated service. Due to advances in technology and logistics industry management of the strategic guiding ideology, supplier responsible for raw material, producers responsible for products and distributors responsible for sales operate logistics by themselves and their relationships became closer and closer. Finally, the integration of logistics service mode occurred. The third party logistics operation mode of the integration embodied the essence and the development level of the modern logistics.

As for the performance evaluation of third party logistics enterprises, many scholars have put forward various evaluation methods and ideas from different aspects and they have made some research achievements

For the current research situation, the evaluation system for the third party logistics enterprises cost from the perspective of the activity cost control has not been fully constructed. This study will improve the performance evaluation index, and expand it based on previous research.

\section{Overview of Logistics Cost Control}

Christopher and Barrett studied on the credibility of task cost method in logistics cost accounting method in. In order to accurately account on logistics cost, Barrett established an analytical framework system of the logistics process for enterprises. The research method of the frame system was task cost approach [3]. Robert V. Delaney the total cost of logistics consists of the inventory holding cost, transportation cost and administrative cost of logistics. This division method provides a reference system for the logistics cost accounting and management [4].In 2014, Pang Jin pointed out 
that the opportunity for cultivation and development of China third party logistics has come [5].Yu Ranjian accounted the activity cost of different processes by applying the benefit antinomy theory and finally he pointed that the logistics cost control should be developed fully according to the theory of logistics integration [6]. Xu Yuqing suggested that the third party logistics enterprise logistics cost is the main part of the corporate cost and the traditional cost methods can calculate its cost[7].Xu Hua put forward the countermeasures to solve the problem of logistics cost: strengthening value chain analysis and management, strengthening cost driver analysis and management, establishing and improving the modern logistics technology system, analyzing and evaluating the internal and external resources condition of enterprises, improving the internal and external resources utilization rate, and paying more attention to the construction of logistics center [8].

Through the above summary it can be found that most of the domestic research literature about logistics cost management focuses on: definition and comparison of activity-based costing and the traditional cost accounting method. The target of the activity-based costing is single. It can only provide reference information for the logistics enterprise from a certain aspect and it goes against the logistics enterprise to control cost information from the fundamental and multi-angle.

\section{Construction of the Performance Evaluation Index System of Cost Control}

Non-financial indicators are about the activity characteristics, namely three aspects: efficiency, time and quality, which are the difference from the traditional cost control methods. Evaluation index system includes 4 first level indicators and 13 level second level indexes. The specific indicators are as follows:

Operation efficiency of the logistics resources (B1)

Including the ratio of logistics activity input and output (work cost / work output) (B11), contract operation capacity utilization rate (used work ability / agreed work ability * 100\%) (B12).

Logistics operation time (B2)

Including repeated work percentage (repeated work time/ total time of completing the work time) (B21), the efficiency of value chain (valid time / task chain cycle) (B22), the rapid and timely rate (promptly complete logistics quantity / total logistics completion quantity * 100\%) (B23).

Logistics operation quality (B3)

Including the work loss rate (total work loss / total operation income in report period) (B31), work accuracy (accurate completion of logistics quantity / total logistics completion quantity *100\%) (B32), works atisfaction (customer satisfaction and worker satisfaction) (B33).

Logistics activity-based costing (B4)

Including value-added operation cost difference (size and the change tendency of value-added operations cost difference) (B41), non value added activity cost control (size and the change tendency of non value added activity cost difference) (B42), improve value-added operation standard (change of value-added operation standard with the new technology, new design and other reform (B43), operation cost rate (all logistics operation cost / total cost of operation * $100 \%$ ) (B44), the unit operation cost rate of turnover (the total operation cost / turnover* 100\%) (B45).

\subsection{Fuzzy Evaluation Method of Operating Cost Control Performance}

Establishment of Evaluation Set. The evaluation set is a collection of evaluation results made for the evaluated objects, which can be expressed as: $V=\left\{v_{1}, v_{2}, \cdots, v_{n}\right\}, V_{k}(k=1,2, \cdots n)$, is the number of possible results of the evaluation. Fuzzy comprehensive evaluation aims to get the best evaluation result from the evaluation set through taking all the influencing factors into consideration. This model uses the evaluation set of five levels. The evaluation set is defined as $V=\left\{v_{1}, v_{2}, v_{3}, v_{4}, v_{5}\right\}$, wherein $v_{1}, v_{2}, v_{3}, v_{4}, v_{5}$ respectively represent "very good", "good", "general", "poor", "very poor".

Determination of the Importance of Each Level Index. This paper combines the subjective weighting with the objective weighting (i.e. the combination weighting method). The first level importance is determined so that the two can have complementary advantages. The second level 
index importance still adopts the subjective weighting method. Subjective weighting uses the hierarchy analysis and the objective weighting uses entropy method.

Determination of Subjective Weighting. According to the methods of experts' opinion, it is determined the weight of activity based cost control four latitude index and its sub factors level index $a$ and $a_{i}$,

$$
\begin{aligned}
& a=\left(a_{1}, a_{2}, a_{3}, a_{4}\right), \sum_{i=1}^{4} a_{i}=1, \\
& a_{i}=\left(a_{i 1}, a_{i 2}, \cdots a_{i m}\right), \sum_{j=1}^{m} a_{i j}=1
\end{aligned}
$$

Among them, $i=1, \ldots, 4 ; j=1, \cdots, m$ ( $a_{i j}$ is the weight of second level index $B_{i j}$ in the primary target in the first level index $B_{i}$ and $\mathrm{m}$ is the number of the second level index).

Determination of the Objective Weight. In order to avoid too much complex weights, this paper chooses the entropy weight method, which mainly determines the level of index weight according to the variation of information index.

In the evaluation problem of $n$ evaluation indexes and $z$ evaluation objects, entropy $i$ evaluation index is defined as

$$
H_{i}=-k \sum_{h=1}^{z} f_{i h} \ln f_{i h}(i=1,2, \cdots, n)
$$

And in the formula $f_{i h}=\frac{l_{\text {ih }}}{\sum_{h=1}^{z} l_{i h}}, k=\frac{1}{\ln z}$ among them $h(1,2, \cdots, z)$ is the evaluation object; $l_{i h} \in[0,1]$ is the first $h$ evaluation object through the standardization of the first index score. And it is assumed that when $f_{i h}=0, f_{i h} \ln f_{i h}=0$. The $i$ entropy evaluation indexes for $\beta_{i}=\frac{1-H_{i}}{n-\sum_{i=1}^{n} H_{i}}$. The objective entropy weight is $\beta=\left(\beta_{1}, \beta_{2}, \cdots \beta_{n}\right)$.

To determine the target combination weight. A more reasonable way of combination of subjective and objective weight is to determine a preference coefficient. The weight after combining the definition is

$W^{\prime}=\mu \times \alpha+(1-\mu) \times \beta$, wherein $a_{i j}$ is the preference coefficient $\mu \in[0,1]$.

After $W^{\prime}$ is normalized, the first level index combined weight is $W=\left(w_{1}, w_{2}, w_{3}, w_{4}\right), \sum_{i=1}^{4}=1$ Expert questionnaire issued in the research is broad, representative, but as the evaluation objects are not very much, namely the third party logistics enterprises using the ABC methods are very few, the subjective preference factor is 0.7 and the objective weight preference coefficient of each take 0.3.

Determination of Second Level Index Combined Weight. The combined weight of the second level index is the first level index $B_{i j}$ is weight $w_{i}$ multiplying the second level index $B_{i j}$ 's weight $a_{i j}$ in the first level index, which is $W_{i j}^{\prime \prime}=w_{i} \times a_{i j}$.

\subsection{Establishment of Judgment Evaluation Set $T$}

Determine the evaluation set of the third party logistics enterprises operating cost control level of performance. The evaluation results are assumed to have five levels, namely the evaluation set

$T=\left\{t_{1}, t_{2}, t_{3}, t_{4}, t_{5}\right\}=\{100,80,60,40,20\}, \quad T c(c=1,2,3,4,5)$ respectively represent excellent, good, medium, poor and very poor. Among them, medium represents the average level of the third party logistics enterprises operating cost control performance.

\subsection{Conducting Second Level Fuzzy Evaluation for Bi}

For each factor of $\mathrm{Bi}$ single factor evaluation is conducted and single factor evaluation matrix 
$R_{i}=\left[\begin{array}{cccc}r_{i 11} & r_{i 12} & \cdots & r_{i 1 c} \\ r_{i 21} & r_{i 22} & \cdots & r_{i 21} \\ \vdots & \vdots & \vdots & \vdots \\ r_{i m 1} & r_{i m 2} & \cdots & r_{i m c}\end{array}\right]$

Wherein, $r_{i m c}$ represents factor's $B_{i j}$ membership grade for evaluation grade $v_{k}$. So through fuzzy comprehensive evaluation for $\mathrm{Bi}$, it gets $B_{i}=a_{i}$.

$R_{i}=\left(a_{i 1}, a_{i 2}, \cdots, a_{i m}\right) \times\left[\begin{array}{cccc}r_{i 11} & r_{i 12} & \cdots & r_{i 1} \\ r_{i 21} & r_{i 22} & \cdots & r_{i 21} \\ \vdots & \vdots & \vdots & \vdots \\ r_{i \text { in1 }} & r_{\text {im2 }} & \cdots & r_{\text {iic }}\end{array}\right]$
$=\left(b_{i 1}, b_{i 2}, \cdots, b_{i c}\right)$

According to the above algorithm, all the evaluation set $B=\left(B_{1}, B_{2}, B_{3}, B_{4}\right)^{T}$.

\subsection{Determination of Fuzzy Evaluation Matrix for First Level Index}

$U=W \bullet B=\left(w_{1}, w_{2}, w_{3}, w_{4}\right) \times\left(B_{1}, B_{2}, B_{3}, B_{4}\right)^{T}$

After the normalization process for $U$, the first level index's fuzzy evaluation matrix

$U^{\prime}=\left(u_{1}, u_{2}, u_{3}, u_{4}, u_{5}\right)$

2.6 Determination of the overall score

$S=U^{\prime} \times T^{T}=\left(u_{1}, u_{2}, u_{3}, u_{4}, u_{5}\right) T^{T}$

\section{Conclusion}

On the basis of the research result of the third party logistics enterprises performance evaluation at home and abroad and in the principal of systematization, feasibility and multi-purposes, this study builds the evaluation index system of the third party logistics enterprise performance and the evaluation model by using combined weight fussy comprehensive evaluation method from the perspective of operating cost control. This method cannot only be used to conduct effective evaluation for the overall performance of the third party logistics enterprises operating cost, but also conduct single examination for a certain aspect of the enterprises. It lets the third party logistics enterprises know the current operating cost control situation and the existing problems, which means the method has the functions of analysis, evaluation and diagnosis. It is hoped that the evaluation method can play a role in making key control means and measurement for improving operating cost of the third party logistics enterprises.

\section{References}

[1] National Standard of the People's Republic of China. Logistics Terminology (GB/T183542001)[S]. Beijing: China Standard Press, 2001.

[2]JIAN Lingxiang, LI Dong-bing. Analysis of using activity-based costing management in third-party logistics enterprises[C]//The 9th Conference of Hong Kong Society for Transportation Studies [J], 2004, 12: 475-481.

[3] Barrett. A New Approach to Logistics Analysis [J]. International Journal of Physical Distribution and Materials Management. 1982:3-26.

[4] Hongren. You Zhanqing, Liu Kesheng, Logistics Economics, Beijing: Mechanical Industry Press, 2007:71.

[5] Pang Jin. The Logistics Cost Analysis and Policy Recommendations [J]. Macroeconomic management, 2014,(8):66-67.

[6] Yu Ranjian .Based on the Method of Third Party Logistics Enterprise Cost Management Research [J].Logistics Engineer and Management, 2014, 36(3):31-33.

[7] Xu Yuqing, Wang Ruijuan, Yang Lujing.The Third Party Logistics Enterprise Logistics Cost Calculation and Cases. Industrial Engineering and Management, 2010, 15(2):56-61.

[8] Xu Hua. Zhang Yanhua. Elementary Analysis of Cost Control of China Third Party Logistics Enterprises [J]. Exploration of Management, 2009, 32(2):30-31. 